\title{
AN ANALOGUE OF A THEOREM OF KURZWEIL
}

\author{
DAVID SIMMONS
}

\begin{abstract}
A theorem of Kurzweil ('55) on inhomogeneous Diophantine approximation states that if $\theta$ is an irrational number, then the following are equivalent: (A) for every decreasing positive function $\psi$ such that $\sum_{q=1}^{\infty} \psi(q)=\infty$, and for almost every $s \in \mathbb{R}$, there exist infinitely many $q \in \mathbb{N}$ such that $\|q \theta-s\|<\psi(q)$, and (B) $\theta$ is badly approximable. This theorem is not true if one adds to condition (A) the hypothesis that the function $q \mapsto q \psi(q)$ is decreasing. In this paper we find a condition on the continued fraction expansion of $\theta$ which is equivalent to the modified version of condition (A). This expands on a recent paper of D. H. Kim ('14).
\end{abstract}

An irrational number $\theta$ is said to be badly approximable (or of bounded type) if there exists $\varepsilon>0$ such that for every rational $p / q \in \mathbb{Q}$,

$$
\left|\theta-\frac{p}{q}\right| \geq \frac{\varepsilon}{q^{2}}
$$

It is well-known that an irrational number $\theta$ is badly approximable if and only if the partial quotients of $\theta$ form a bounded sequence. Another equivalent condition was given by Kurzweil [6]. To state it, let us define the set

$$
W(\theta, \psi)=\left\{s \in \mathbb{R}: \exists^{\infty} q \in \mathbb{N}\|q \theta-s\|<\psi(q)\right\},
$$

where $\|\cdot\|$ denotes distance to the nearest integer. Then Kurzweil's result may be stated as follows: $\theta$ is badly approximable if and only if for every decreasing function $\psi: \mathbb{N} \rightarrow(0, \infty)$ such that $\sum_{q=1}^{\infty} \psi(q)=\infty$, the set $W(\theta, \psi)$ has full measure. (Note that if $\sum_{q=1}^{\infty} \psi(q)<\infty$, then the set $W(\theta, \psi)$ has measure zero by the Borel-Cantelli lemma.)

Rather than considering all decreasing functions $\psi$, one may consider the smaller class of Khinchin sequences: a function $\psi: \mathbb{N} \rightarrow(0, \infty)$ is called a Khinchin sequence if, in addition to the divergence condition $\sum_{q=1}^{\infty} \psi(q)=\infty$, the function $q \mapsto q \psi(q)$ is nonincreasing. Although less natural than the condition that $\psi$ is decreasing, the hypothesis that a sequence is a Khinchin sequence is significant both for historical reasons (Khinchin first proved his eponymous theorem [3] in the setting of Khinchin sequences, although his theorem was later generalized) and because such sequences are often easier to work with.

Let $\theta$ be an irrational number and let $\psi$ be a Khinchin sequence. A recent paper of D. H. Kim 5 gives a criterion, based on the continued fraction expansion of $\theta$, for the set $W(\theta, \psi)$ to have full measure 1 However, his paper leaves open the question of finding an analogue of Kurzweil's theorem in the setting of Khinchin sequences, although he proves several results in that direction [5, §3]. The aim of this paper is to complete the work of Kim by proving such an analogue.

Acknowledgements. The author thanks Lior Fishman for helpful comments.

\section{Statement OF Results}

We first recall the main theorem of [5], rephrased slightly 2

Theorem 1.1 ([5. Theorem 2.1]). Fix $\theta \in \mathbb{R} \backslash \mathbb{Q}$ and let $\left(q_{k}\right)_{0}^{\infty}$ be the sequence of the denominators of the convergents of $\theta$. Let $\psi: \mathbb{N} \rightarrow(0, \infty)$ be a Khinchin sequence, and let $\phi(q)=1 /(q \psi(q))$. Then the following are equivalent:

\footnotetext{
${ }^{1}$ After this paper was written, Kim extended his result to all positive decreasing sequences in a joint paper with M. Fuchs [2].

${ }^{2}$ Technically, the result of [5] applies to the sets $\bigcap_{\varepsilon>0} W(\theta, \varepsilon \psi)$ and not directly to the sets $W(\theta, \psi)$. But since the convergence or divergence of the series (1.1) is invariant under a slight perturbation of $\psi$, [5] Theorem 2.1] and Theorem 1.1 are equivalent.
} 
(A) $W(\theta, \psi)$ has full measure.

(B) The series

$$
\sum_{k=0}^{\infty} \frac{\log \phi\left(q_{k}\right) \wedge \log \left(q_{k+1} / q_{k}\right)}{\phi\left(q_{k}\right)}
$$

diverges. (In this paper, $\wedge$ and $\vee$ denote minimum and maximum, respectively.)

To state our main theorem, we use the notation

$$
\Sigma\left(\left(a_{i}\right)_{1}^{n}: m\right)
$$

to denote the sum of the $m$ largest elements of the sequence $\left(a_{i}\right)_{1}^{n}$, with $\Sigma\left(\left(a_{i}\right)_{1}^{n}: m\right)=\sum_{1}^{n} a_{i}$ if $m \geq n$. For $\alpha \geq 0$, we let $\Sigma\left(\left(a_{i}\right)_{1}^{n}: \alpha\right)=\Sigma\left(\left(a_{i}\right)_{1}^{n}:\lfloor\alpha\rfloor\right)$.

Theorem 1.2. Fix $\theta \in \mathbb{R} \backslash \mathbb{Q}$ and let $\left(q_{k}\right)_{0}^{\infty}$ be the sequence of the denominators of the convergents of $\theta$. Then the following are equivalent:

(A) For every Khinchin sequence $\psi: \mathbb{N} \rightarrow(0, \infty)$, the set $W(\theta, \psi)$ has full measure.

(B) For some $\varepsilon>0$,

$$
\limsup _{k \rightarrow \infty} \frac{1}{\log \left(q_{k}\right)} \Sigma\left(\left(\log \left(\frac{q_{i+1}}{q_{i}}\right)\right)_{i=0}^{k-1}: \frac{\varepsilon \log \left(q_{k}\right)}{\log \log \left(q_{k}\right)}\right)<1 .
$$

Remark. Since condition (B) of Theorem 1.2 is not equivalent to the condition that the sequence $\left(q_{k}\right)_{1}^{\infty}$, it follows from Kurzweil's theorem that condition $(\mathrm{A})$ is not equivalent to the condition that for every decreasing positive function $\psi: \mathbb{N} \rightarrow(0, \infty)$ such that $\sum_{q=1}^{\infty} \psi(q)=\infty$, the set $W(\theta, \psi)$ has full measure. In particular, there exists a decreasing positive function $\psi: \mathbb{N} \rightarrow(0, \infty)$ such that $\sum_{q=1}^{\infty} \psi(q)=\infty$ and such that there is no Khinchin sequence $\psi^{\prime}: \mathbb{N} \rightarrow(0, \infty)$ with $\psi^{\prime}(q) \leq \psi(q)$ for all $q$. An example of such a sequence is given by the formula

$$
\psi(q)=2^{-n_{k}} \quad\left(2^{n_{k-1}} \leq q<2^{n_{k}}\right)
$$

where $\left(n_{k}\right)_{1}^{\infty}$ is any sequence of integers such that $n_{k}-n_{k-1} \geq k$ for all $k$.

\section{Proof of Theorem 1.2}

Convention. The symbol $\asymp$ will denote a coarse multiplicative asymptotic, i.e. $A_{n} \asymp B_{n}$ means that there exists a constant $C>0$ (the implied constant) such that $C^{-1} B_{n} \leq A_{n} \leq C B_{n}$.

Proof of $(\mathrm{A}) \Rightarrow(\mathrm{B})$. By contradiction, suppose that (B) is false. Then for each $n \in \mathbb{N}$, there exists $k_{n} \in \mathbb{N}$ such that

$$
\frac{1}{\log \left(q_{k_{n}}\right)} \Sigma\left(\left(\log \left(\frac{q_{i+1}}{q_{i}}\right)\right)_{i=0}^{k_{n}-1}: \frac{1}{2^{n}} \frac{\log \left(q_{k_{n}}\right)}{\log \log \left(q_{k_{n}}\right)}\right) \geq 1-\frac{1}{2^{n}} .
$$

Without loss of generality, suppose that the sequence $\left(k_{n}\right)_{1}^{\infty}$ is increasing, and let $k_{0}=0$. For each $n \geq 1$, let $S_{n}^{\prime}$ be a subset of $\left\{0, \ldots, k_{n}-1\right\}$ of cardinality at most $\frac{1}{2^{n}} \frac{\log \left(q_{k_{n}}\right)}{\log \log \left(q_{k_{n}}\right)}$ such that

$$
\sum_{k \in S_{n}^{\prime}} \log \left(\frac{q_{k+1}}{q_{k}}\right) \geq\left(1-2^{-n}\right) \log \left(q_{k_{n}}\right) .
$$

Then let $S_{n}=S_{n}^{\prime} \backslash\left\{0, \ldots, k_{n-1}-1\right\}$ and $T_{n}=\left\{k_{n-1}, \ldots, k_{n}-1\right\} \backslash S_{n}$. Then

$$
\#\left(S_{n}\right) \leq \frac{1}{2^{n}} \frac{\log \left(q_{k_{n}}\right)}{\log \log \left(q_{k_{n}}\right)}
$$

and

$$
\sum_{k \in T_{n}} \log \left(\frac{q_{k+1}}{q_{k}}\right) \leq \log \left(q_{k_{n}}\right)-\sum_{k \in S_{n}^{\prime}} \log \left(\frac{q_{k+1}}{q_{k}}\right) \leq 2^{-n} \log \left(q_{k_{n}}\right)
$$


Now define the function $\phi: \mathbb{N} \rightarrow(0, \infty)$ by the formula

$$
\phi(q)=\log \left(q_{k_{n}}\right) \forall q_{k_{n-1}} \leq q<q_{k_{n}} .
$$

Then $\phi$ is nondecreasing, and

$$
\begin{aligned}
\sum_{q=1}^{\infty} \frac{1}{q \phi(q)} & =\sum_{n=1}^{\infty} \frac{1}{\log \left(q_{k_{n}}\right)} \sum_{q=q_{k_{n}-1}}^{q_{k_{n}}-1} \frac{1}{q} \asymp \sum_{n=1}^{\infty} \frac{\log \left(q_{k_{n}} / q_{k_{n-1}}\right)}{\log \left(q_{k_{n}}\right)} \\
& =\sum_{n=1}^{\infty}\left[1-\frac{\log \left(q_{k_{n-1}}\right)}{\log \left(q_{k_{n}}\right)}\right] \asymp \sum_{n=1}^{\infty} 1 \wedge \log \left(\frac{\log \left(q_{k_{n}}\right)}{\log \left(q_{k_{n-1}}\right)}\right)=\infty .
\end{aligned}
$$

Thus $\psi(q)=1 /(q \phi(q))$ is a Khinchin sequence. So by (A) together with Theorem 1.1] the series (1.1) diverges. On the contrary, we show that (1.1) converges:

$$
\begin{aligned}
& \sum_{k=0}^{\infty} \frac{\log \phi\left(q_{k}\right) \wedge \log \left(q_{k+1} / q_{k}\right)}{\phi\left(q_{k}\right)} \\
& \leq \sum_{n=1}^{\infty}\left[\sum_{k \in S_{n}} \frac{\log \phi\left(q_{k}\right)}{\phi\left(q_{k}\right)}+\sum_{k \in T_{n}} \frac{\log \left(q_{k+1} / q_{k}\right)}{\phi\left(q_{k}\right)}\right] \\
& =\sum_{n=1}^{\infty}\left[\frac{\log \log \left(q_{k_{n}}\right)}{\log \left(q_{k_{n}}\right)} \#\left(S_{n}\right)+\frac{1}{\log \left(q_{k_{n}}\right)} \sum_{k \in T_{n}} \log \left(q_{k+1} / q_{k}\right)\right] \\
& \leq \sum_{n=1}^{\infty}\left[\frac{1}{2^{n}}+\frac{1}{2^{n}}\right] \\
& =2<\infty .
\end{aligned}
$$

This contradiction completes the proof.

Proof of $(\mathrm{B}) \Rightarrow(\mathrm{A})$. Let $\psi: \mathbb{N} \rightarrow \infty$ be a Khinchin sequence, and by contradiction suppose that $W(\theta, \psi)$ does not have full measure. Then by Theorem 1.1 the series (1.1) converges, where $\phi(q)=1 /(q \psi(q))$ is nondecreasing. Let

so that

$$
S=\left\{k: \phi\left(q_{k}\right) \leq q_{k+1} / q_{k}\right\}, T=\mathbb{N} \backslash S,
$$

$$
\infty>\sum_{k=0}^{\infty} \frac{\log \phi\left(q_{k}\right) \wedge \log \left(q_{k+1} / q_{k}\right)}{\phi\left(q_{k}\right)}=\sum_{k \in S} \frac{\log \phi\left(q_{k}\right)}{\phi\left(q_{k}\right)}+\sum_{k \in T} \frac{\log \left(q_{k+1} / q_{k}\right)}{\phi\left(q_{k}\right)} .
$$

For each $m \in \mathbb{N}$, let $Q_{m}$ be the largest integer such that $\phi\left(Q_{m}\right) \leq 2^{m}$. Then

$$
\begin{aligned}
\frac{1}{\phi(q)} \asymp \sum_{m \in \mathbb{N}: 2^{m} \geq \phi(q)} \frac{1}{2^{m}} & =\sum_{m \in \mathbb{N}: q \leq Q_{m}} \frac{1}{2^{m}} \\
\frac{\log \phi(q)}{\phi(q)} \asymp \sum_{m \in \mathbb{N}: 2^{m} \geq \phi(q)} \frac{m}{2^{m}} & =\sum_{m \in \mathbb{N}: q \leq Q_{m}} \frac{m}{2^{m}}
\end{aligned}
$$

and thus

$$
\begin{aligned}
\infty & =\sum_{q=1}^{\infty} \frac{1}{q \phi(q)} \asymp \sum_{m=0}^{\infty} \sum_{q=1}^{Q_{m}} \frac{1}{q 2^{m}} \asymp \sum_{m=0}^{\infty} \frac{\log \left(Q_{m}\right)}{2^{m}} \\
\infty & >\sum_{k \in S} \frac{\log \phi\left(q_{k}\right)}{\phi\left(q_{k}\right)}+\sum_{k \in T} \frac{\log \left(q_{k+1} / q_{k}\right)}{\phi\left(q_{k}\right)} \\
& \asymp \sum_{m=0}^{\infty}\left[\frac{m}{2^{m}} \#\left\{k \in S: q_{k} \leq Q_{m}\right\}+\frac{1}{2^{m}} \sum_{\substack{k \in T \\
q_{k} \leq Q_{m}}} \log \left(\frac{q_{k+1}}{q_{k}}\right)\right] .
\end{aligned}
$$


It follows that if

$$
\begin{aligned}
\lambda_{m}= & \frac{\frac{m}{2^{m}} \#\left\{k \in S: q_{k} \leq Q_{m}\right\}+\frac{1}{2^{m}} \sum_{\substack{k \in T \\
q_{k} \leq Q_{m}}} \log \left(\frac{q_{k+1}}{q_{k}}\right)}{\frac{\log \left(Q_{m}\right)}{2^{m}}} \\
= & \frac{1}{\log \left(Q_{m}\right)}\left[m \#\left\{k \in S: q_{k} \leq Q_{m}\right\}+\sum_{\substack{k \in T \\
q_{k} \leq Q_{m}}} \log \left(\frac{q_{k+1}}{q_{k}}\right)\right],
\end{aligned}
$$

then

On the other hand, if

$$
\liminf _{m \rightarrow \infty} \lambda_{m}=0
$$

then

$$
\kappa_{m}=\frac{m}{2^{m}} \#\left\{k \in S: q_{k} \leq Q_{m}\right\}
$$

$$
\lim _{m \rightarrow \infty} \kappa_{m}=0
$$

Fix $\varepsilon>0$, and choose $m \geq 2$ such that $\lambda_{m}, \kappa_{m} \leq \varepsilon$. Then

Consider the function

$$
\frac{m}{2^{m}} \vee \frac{m}{\log \left(Q_{m}\right)} \leq \frac{\varepsilon}{\#\left\{k \in S: q_{k} \leq Q_{m}\right\}}
$$

$$
f(x)=\frac{x}{2^{x}} \vee \frac{x}{\log \left(Q_{m}\right)} \quad(x \geq 2) .
$$

Since $f$ is the maximum of an increasing function and a decreasing function, $f$ has a unique minimum, which occurs when the two inputs to the maximum agree, namely at $x=\log _{2} \log \left(Q_{m}\right)$. Thus

$$
\frac{\varepsilon}{\#\left\{k \in S: q_{k} \leq Q_{m}\right\}} \geq f(m) \geq \min (f)=\frac{\log _{2} \log \left(Q_{m}\right)}{\log \left(Q_{m}\right)} \geq \frac{\log \log \left(Q_{m}\right)}{\log \left(Q_{m}\right)}
$$

i.e.

$$
\#\left\{k \in S: q_{k} \leq Q_{m}\right\} \leq \frac{\varepsilon \log \left(Q_{m}\right)}{\log \log \left(Q_{m}\right)} .
$$

On the other hand, since $\lambda_{m} \leq \varepsilon$,

$$
\sum_{\substack{k \in T \\ q_{k} \leq Q_{m}}} \log \left(\frac{q_{k+1}}{q_{k}}\right) \leq \varepsilon \log \left(Q_{m}\right)
$$

Let $k_{m}$ be the smallest integer such that $Q_{m}<q_{k_{m}}$. Then

$$
\begin{gathered}
\#\left\{k \in S: k<k_{m}\right\} \leq \frac{\varepsilon \log \left(q_{k_{m}}\right)}{\log \log \left(q_{k_{m}}\right)} \\
\sum_{\substack{k \in T \\
k<k_{m}}} \log \left(\frac{q_{k+1}}{q_{k}}\right) \leq \varepsilon \log \left(q_{k_{m}}\right)
\end{gathered}
$$

and thus

$$
\Sigma\left(\left(\log \left(\frac{q_{i+1}}{q_{i}}\right)\right)_{i=0}^{k_{m}-1}: \frac{\varepsilon \log \left(q_{k_{m}}\right)}{\log \log \left(q_{k_{m}}\right)}\right) \geq(1-\varepsilon) \log \left(q_{k_{m}}\right) .
$$

Since $\varepsilon$ was arbitrary and $k_{m} \rightarrow \infty$, for all $\varepsilon>0$ we have

$$
\limsup _{k \rightarrow \infty} \frac{1}{\log \left(q_{k}\right)} \Sigma\left(\left(\log \left(\frac{q_{i+1}}{q_{i}}\right)\right)_{i=0}^{k-1}: \frac{\varepsilon \log \left(q_{k}\right)}{\log \log \left(q_{k}\right)}\right)=1,
$$

contradicting $(\mathrm{B})$. 


\section{Consequences of Theorem 1.2}

In this section we use Theorem 1.2 to prove some necessary and sufficient conditions on $\theta$ for $W(\theta, \psi)$ to be full measure for every Khinchin sequence $\psi$, including reproving some results from [5, §3]. For convenience let

$$
\Omega=\{\theta \in \mathbb{R}: \text { for every Khinchin sequence } \psi \text {, the set } W(\theta, \psi) \text { has full measure }\} .
$$

In other words, $\Omega$ is the set of all $\theta$ such that the equivalent conditions of Theorem 1.2 hold.

Theorem 3.1. Fix $\theta \in \mathbb{R} \backslash \mathbb{Q}$ and let $\left(q_{k}\right)_{0}^{\infty}$ be the sequence of the denominators of the convergents of $\theta$.

(i) If

$$
\limsup _{k \rightarrow \infty} \frac{\log \left(q_{k}\right)}{k}<\infty
$$

then $\theta \in \Omega$.

(ii) If

$$
\limsup _{k \rightarrow \infty} \frac{\log \left(q_{k}\right)}{k \log (k)}=\infty
$$

then $\theta \notin \Omega$.

(iii) If

$$
\sum_{k=2}^{\infty} \frac{1}{\log \left(q_{k}\right)}<\infty
$$

then $\theta \notin \Omega$.

(iv) If

$$
\limsup _{k \rightarrow \infty} \frac{q_{k+1} / q_{k}}{\log \left(q_{k}\right)}<\infty
$$

then $\theta \in \Omega$.

(v) If

$$
\limsup _{k \rightarrow \infty} \frac{\log \left(q_{k+1} / q_{k}\right)}{\log \left(q_{k}\right)}=\infty
$$

then $\theta \notin \Omega$.

Remark. Parts (i), (iii), and (iv) correspond to [5, Theorem 3.1 and Proposition 3.2]. Although in some cases the new proofs are not shorter than the old proofs, having two proofs may bring further insight.

Remark. By well-known facts about continued fractions (e.g. [4, Theorems 9 and 13]), the conditions (3.4) and (3.5) have interpretations in terms of Diophantine approximation:

- $\theta$ satisfies (3.4) if and only if for some $\varepsilon>0, \theta$ is not $\psi$-approximable, where

$$
\psi(q)=\frac{\varepsilon}{q^{2} \log (q)} .
$$

We recall that a number $\theta$ is called $\psi$-approximable if there exist infinitely many rationals $p / q \in \mathbb{Q}$ such that

$$
\left|\theta-\frac{p}{q}\right|<\psi(q) .
$$

- $\theta$ satisfies (3.5) if and only if $\theta$ is a Liouville number. We recall that a number $\theta$ is called Liouville if for all $n \in \mathbb{N}, \theta$ is $\psi_{n}$-approximable, where $\psi_{n}(q)=q^{-n}$.

Remark. Any badly approximable number $\theta$ satisfies both (3.1) and (3.4), so BA $\subseteq \Omega$. This can also be seen from Kurzweil's theorem.

Remark. The continued fraction expansion of $e$ (see e.g. [1]) satisfies (3.4), so $e \in \Omega$. 
Proof of (i). Choose $M<\infty$ so that for all $k, \log \left(q_{k}\right) \leq M k$. Let $\varepsilon>0$ be arbitrary (e.g. $\left.\varepsilon=1\right)$. Then for sufficiently large $k$,

$$
\frac{\varepsilon \log \left(q_{k}\right)}{\log \log \left(q_{k}\right)} \leq \frac{\varepsilon M k}{\log (M k)} \leq \frac{k}{8}
$$

Let $S \subseteq\{0, \ldots, k-1\}$ be a subset of cardinality at most $k / 8$, and let $T=\{0, \ldots, k-1\} \backslash S$. A counting argument shows that

and thus

$$
\#\{i=0, \ldots, k-1 \text { even }: i, i+1 \in T\} \geq k / 4 \text {, }
$$

$$
\sum_{i \in T} \log \left(q_{i+1} / q_{i}\right) \geq \sum_{\substack{i \text { even } \\ i, i+1 \in T}} \log \left(q_{i+2} / q_{i}\right) \geq(k / 4) \log (2) \geq \frac{\log (2)}{4 M} \log \left(q_{k}\right)
$$

It follows that

$$
\frac{1}{\log \left(q_{k}\right)} \Sigma\left(\left(\log \left(\frac{q_{i+1}}{q_{i}}\right)\right)_{i=0}^{k-1}: \frac{\varepsilon \log \left(q_{k}\right)}{\log \log \left(q_{k}\right)}\right) \leq 1-\frac{\log (2)}{4 M} .
$$

To complete the proof, we take the limsup as $k \rightarrow \infty$ and then apply Theorem 1.2 .

Proof of (ii). Fix $\varepsilon>0$. By assumption, there exist infinitely many $k$ satisfying

$$
\log \left(q_{k}\right) \geq \frac{2}{\varepsilon} k \log (k)
$$

For such $k$,

$$
\frac{\varepsilon \log \left(q_{k}\right)}{\log \log \left(q_{k}\right)} \geq \frac{\varepsilon(2 / \varepsilon) k \log (k)}{\log ((2 / \varepsilon) k \log (k))} \geq \frac{2 k \log (k)}{\log \left(k^{2}\right)}=k,
$$

where the middle inequality holds for all $k$ sufficiently large. But then

$$
\Sigma\left(\left(\log \left(\frac{q_{i+1}}{q_{i}}\right)\right)_{i=0}^{k-1}: \frac{\varepsilon \log \left(q_{k}\right)}{\log \log \left(q_{k}\right)}\right)=\sum_{i=0}^{k-1} \log \left(\frac{q_{i+1}}{q_{i}}\right)=\log \left(q_{k}\right) .
$$

To complete the proof, we divide by $\log \left(q_{k}\right)$, take the limsup as $k \rightarrow \infty$, and apply Theorem 1.2 .

Since (3.3) implies (3.2), (iii) does not require a separate proof.

Proof of (iv). Choose $M<\infty$ such that for all $k, q_{k+1} / q_{k} \leq M \log \left(q_{k}\right)$. Then for all $\varepsilon>0$ and $k \in \mathbb{N}$,

$$
\begin{aligned}
\Sigma\left(\left(\log \left(\frac{q_{i+1}}{q_{i}}\right)\right)_{i=0}^{k-1}: \frac{\varepsilon \log \left(q_{k}\right)}{\log \log \left(q_{k}\right)}\right) & \leq \frac{\varepsilon \log \left(q_{k}\right)}{\log \log \left(q_{k}\right)} \max \left\{\log \left(\frac{q_{i+1}}{q_{i}}\right): i=0, \ldots, k-1\right\} \\
& \leq \frac{\varepsilon \log \left(q_{k}\right)}{\log \log \left(q_{k}\right)} \log \left(M \log \left(q_{k}\right)\right) \leq 2 \varepsilon \log \left(q_{k}\right),
\end{aligned}
$$

where the last inequality holds for all $k$ large enough such that $q_{k} \geq e^{M}$. To complete the proof, we let $\varepsilon=1 / 4$, divide by $\log \left(q_{k}\right)$, take the limsup as $k \rightarrow \infty$, and apply Theorem 1.2 .

Proof of (v). The assumption (3.5) implies that

$$
\limsup _{k \rightarrow \infty} \frac{\log \left(q_{k} / q_{k-1}\right)}{\log \left(q_{k}\right)}=1 .
$$

Fix $\varepsilon>0$. By assumption, there exist infinitely many $k$ such that

$$
\log \left(q_{k} / q_{k-1}\right) \geq(1-\varepsilon) \log \left(q_{k}\right) .
$$

For such $k$, if we assume that $k$ is chosen large enough so that $\frac{\varepsilon \log \left(q_{k}\right)}{\log \log \left(q_{k}\right)} \geq 1$, then

$$
\Sigma\left(\left(\log \left(\frac{q_{i+1}}{q_{i}}\right)\right)_{i=0}^{k-1}: \frac{\varepsilon \log \left(q_{k}\right)}{\log \log \left(q_{k}\right)}\right) \geq \log \left(\frac{q_{k}}{q_{k-1}}\right) \geq(1-\varepsilon) \log \left(q_{k}\right) .
$$

To complete the proof, we divide by $\log \left(q_{k}\right)$, take the limsup as $k \rightarrow \infty$, use the fact that $\varepsilon$ was arbitrary, and apply Theorem 1.2 


\section{REFERENCES}

1. H. Cohn, A short proof of the simple continued fraction expansion of e, Amer. Math. Monthly 113 (2006), no. 1, 57-62.

2. M. Fuchs and D. H. Kim, On Kurzweil's 0-1 law in inhomogeneous Diophantine approximation, http://arxiv.org/abs/1501.04714 preprint 2015.

3. A. Y. Khinchin, Einige Sätze über Kettenbrüche, mit Anwendungen auf die Theorie der Diophantischen Approximationen, Math. Ann. 92 (1924), 115-125 (German).

4. Continued fractions, The University of Chicago Press, Chicago, Ill.-London, 1964.

5. D. H. Kim, Refined shrinking target property of rotations, Nonlinearity 27 (2014), no. 9, 1985-1997.

6. J. Kurzweil, On the metric theory of inhomogeneous diophantine approximations, Studia Math. 15 (1955), 84-112.

Ohio State University, Department of Mathematics, 231 W. 18th Avenue, Columbus, OH 43210-1174, USA

E-mail address: simmons.465@osu.edu

$U R L:$ https://sites.google.com/site/davidsimmonsmath/ 